\title{
DAME SÉBASTIENNE ET LE CHRIST FLÉCHÉ. ICONOGRAPHIE EI MÉMOIRE RITUELLE: LE CAS DU NOUVEAU-MEXIQUE
}

\author{
Carlo Severi \\ École des Hautes Etudes en Sciences Sociales - France
}

Résumé: Au début du XIXe siècle, au Nouveau-Mexique, la nécessité de célébrer un certain nombre de rituels indispensables à la vie d'une communauté catholique, a donné naissance à une tradition religieuse nouvelle, qui a longtemps échappé au contrôle de l'Eglise. Cet article analyse la naissance de cette tradition et in particulier le type d'invention iconographique qui l'a caractérisée. L'invention la plus remarquable a été sans doute une représentation de la mort, nommée Dona Sebastiana. En montrant que cette représentation est l'image intense d'un conflit latent avec les Indiens, cet article propose une thèse d'ordre plus général: les iconographies peuvent représenter le conflit culturel à travers le paradoxe visuel.

Mots-clé: Dame Sébastienne, iconografhie et mort, image e mémoire, Nouveau-Mexique.

Resumo: No começo do século XIX, na região do Novo México, a necessidade de celebrar alguns rituais essenciais, num contexto definido não apenas pelo isolamento, mas também pelo constante contato/conflito com os índios, fez nascer uma nova tradição religiosa, quase completamente fora de qualquer controle eclesiástico. Este paper é um estudo dessas novas tradições religiosas e de sua iconografia. A mais importante invenção iconográfica desta religião é a representação da Morte, chamada de Dona Sebastiana. Evidenciando que Sebastiana é uma poderosa imagem de conflitos não resolvidos com os índios, este paper postula que iconografia ritual pode representar conflito cultural através de paradoxos visuais.

Palavras-chave: Dona Sebastiana, iconografia e morte, imagem e memória, Novo México.

L'histoire des régions qu'on appelle aujourd'hui Arizona et NouveauMexique, depuis la Conquête jusqu'à la deuxième moitié du XIX ${ }^{\mathrm{e}}$ siècle est connue: un conflit intense entre Indiens et populations venus du Mexique, des 
attaques incessantes des Indiens à des communautés néo hispaniques isolées et très pauvres, des ripostes parfois sanglantes mais toujours discontinues de la part des défenseurs des Missions. Cette histoire, qui va environ de la rébellion des Zuni en 1635, à celles des Hopi en 1680, jusqu'à la soumission forcée des Apaches de l'Ouest en 1870, et à l'occupation Nord-américaine de ces régions, a été retracée par un certain nombre d'historiens et d'anthropologues (Marta Weigle, George Kubler ou George Goodwin pour les Apaches).

Pour entamer notre analyse des iconographies néo-hispaniques, nous ne soulignerons qu'un seul point: avec l'extinction graduelle de la prédication franciscaine, il se produit dans la région, auprès des populations d'origine hispaniques, à partir de la fin du XVIII ${ }^{e}$ siècle, une carence exceptionnelle, reconnue par tous, de personnel ecclésiastique.avec l'extinction graduelle de la prédication franciscaine, la région se trouve victime, à partir de la fin du XVIII ${ }^{\mathrm{e}}$ siècle, d'une carence unanimement reconnue, de personnel ecclésiastique. Dès la proclamation de l’Indépendance du Mexique, en 1821, le clergé néomexicain se retire de la région et tend à revenir en Espagne (Stark, 1971, p. 304). Il se crée, ainsi, une situation vraiment exceptionnelle qui met en péril la survie même des communautés de langue espagnole, où il devient impossible de célébrer les rituels essentiels scandant le cycle de vie d'un chrétien: le baptême, l'eucharistie, les mariages, les rituels funéraires. Antonio Barreiro, un juriste qui se rend au Nouveau-Mexique en 1831, note dans l'une de ses lettres ceci:

L’administration spirituelle se trouve dans un état vraiment lamentable. Rien n'est plus commun que de voir un grand nombre de malades mourir sans confession ni extrême-onction. Rien n'est plus rare que de voir l'administration de l'eucharistie. Les cadavres restent sans sépulture pendant de longs jours. Les nouveau-nés ne sont baptisés que rarement et au prix de mille sacrifices. Il existe ici un nombre considérable de malheureux qui doivent se dispenser d'aller à la messe pendant la plus grande partie de l'année. Les églises sont presque toutes détruites et la majorité d'entre elles ne sont certainement pas dignes de s'appeler temples de Dieu. (Escudero, 1971, p. 18).

Les chiffres, établis par les recherches historiques de Marta Weigle (1977a), sont éloquents. Dans toute la région du Nouveau-Mexique, qui compte en 1850 environ 60000 habitants dont un tiers d'Indiens opèrent vingt-deux franciscains, en 1776, trente en 1788, vingt-deux franciscains et deux prêtres en 1812, neuf franciscains et quatre prêtres en 1826. En 1828, il reste seulement deux 
franciscains. Lorsque l'évêque français de Santa Fe, récemment nommé, arrive dans sa ville, il ne peut compter pour la reconquête spirituelle de la région que sur une dizaine de prêtres.

De la nécessité de célébrer les rituels essentiels, dans une situation définie non seulement par l'isolement, mais aussi par un contact et des conflits constants avec les Indiens, naît ainsi une nouvelle tradition religieuse, échappant presque au contrôle ecclésiastique. C'est dans cette situation que peut naître la principale organisation religieuse des communautés néomexicaines, une association qui, sans se proclamer jamais nouvelle Église, va introduire des modifications remarquables et radicales dans le culte du Christ et va défendre jalousement son autonomie par rapport à la hiérarchie ecclésiastique. Il s’agit de la Cofradía de los Hermanos del Santo Sangre, dite aussi Hermandad de los Penitentes, une confrérie rurale de secours mutuel qui, dès la seconde moitié du XvIII ${ }^{\mathrm{e}}$ siècle peut-être, se répand très rapidement dans une vaste région comprise entre la frontière actuelle Mexique-États-Unis et le nord du Rio Grande (NouveauMexique). Au sein de ces sociétés religieuses, dont on commence à avoir des témoignages fiables autour de 1810, naissent de nouvelles églises non consacrées par le clergé: les moradas. Celles-ci deviennent le lieu où de nouvelles images religieuses, ainsi que de nouveaux rituels, s'établissent. Les cultes des moradas célébrés par les Penitentes ont un tel succès que l'Église catholique, pendant tout le XIX ${ }^{\mathrm{e}}$ siècle et jusqu'aux années 1950 (Weigle, 1977a, p. 11), va peiner pour imposer aux communautés néomexicaines des statuts plus proches de l'orthodoxie de Rome et des rituels respectueux de sa tradition.

La Confrérie néomexicaine s’organise en apparence comme une confrérie religieuse européenne classique, aux traits fortement andalous. Elle en imite, d'ailleurs, bien des traits. Comme d'autres associations analogues, elle a surtout un caractère initiatique et se trouve fondée, du point de vue sociologique, sur la distinction entre les Hermanos de Sangre, les Frères de sang et véritables Pénitents, et les Hermanos de la Luz, les Frères de la lumière, qui jouent plutôt un rôle d'organisation et de direction spirituelle (Weigle, 1977a; Weigle; Lyons, 1982). Comme dans la Confrérie de Notre-Père Jésus de Séville, dans laquelle Chavez (1954) et Woodward (1975) ont identifié l'une des origines probables des Penitentes américains, la vie religieuse de la communauté est centrée sur l'exercice de la pénitence ainsi que sur l'imitation du Christ. En particulier, le pénitent membre de la confrérie est toujours tenu de pratiquer l'autoflagellation au cours des processions de la Semaine sainte. Toutefois, les différences entre les cultes néomexicains et les usages européens ne tardent pas à apparaître et 
à susciter l'alarme de l'Église. Les aspects nouveaux sont essentiellement au nombre de deux. Le premier consiste en un cycle rituel consacré à la célébration de la Passion, où toutes les phases du martyre du Christ (flagellation à la colonne, chemin de Croix et Crucifixion) sont fidèlement reproduites. La célébration rituelle de la Passion, en particulier, prend parmi les Pénitents la forme violente d'une Crucifixion simulée. Le second aspect, troublant pour l'Église, consiste en une curieuse représentation de la mort, qui apparaît bientôt sur les autels des chapelles secrètes de la Cofradía réservées à ses seuls membres. Ces innovations se différencient nettement de la tradition catholique mexicaine ou espagnole. Elles suscitent, d'une part, un respect mêlé à une certaine crainte auprès des paysans et, d'autre part, le scandale dans le clergé. Elles procurent aussi à la Confrérie l'hostilité ouverte et pugnace de la hiérarchie ecclésiastique, qui parle alors non seulement d'excès et de péchés, mais aussi parfois de véritables crimes et de croyances hérétiques, jugés même analogues à celles des Indiens (Rieupeyrout, 1987, p. 52-53). Au cours de la campagne conduite avec ténacité contre les Penitentes on ira jusqu'à affirmer qu'ils «adoraient la mort comme les Indiens» pendant leurs processions. On déclarera aussi que dans l'intention de simuler la crucifixion pendant la Semaine sainte, ils faisaient subir à de jeunes membres de la confrérie de véritables tortures: ainsi, disait-on, ils les contraignaient à marcher en portant d'énormes croix en bois sur leur dos; puis ils les liaient et peut-être même les clouaient sur l'une de ces croix, imitation du martyre de Jésus-Christ. Déjà en 1833, l'évêque de Durango, Zubiria, lançait l'alarme et interdisait dans une lettre pastorale très sévère l'exercice de ces nouveaux rituels. Le ton de Zubiria était grave, presque menaçant:

J'interdis ces fraternités de pénitence ou plutôt de boucherie qui ont poussé, protégées par une tolérance coupable [...] et qu'on recueille en aucun village ces grands troncs d'arbre [...] avec lesquels on tue les corps et en même temps néglige l'âme, en la laissant des années entières dans la faute [...]. (Weigle, 1977a, p. 195-196).

Ces admonestations restèrent sans écho. En 1879 l'évêque français de Santa Fe, Jean-Baptiste Lamy, qui avait conduit une inspection très soignée dans tout le territoire, suivie par plusieurs menaces d'excommunication, pouvait encore écrire que ces rituels excessifs continuaient, et même que certains des jeunes choisis pour représenter la Passion sur la croix étaient morts pendant le rituel: 
Malheureusement, les chefs de ces sociétés n’obéissent pas à nos ordres. Autrefois, ils ont subi eux-mêmes ces pratiques et coutumes cruelles, dans des lieux retirés, la nuit, en s’infligeant eux-mêmes des coups de fouet si terribles qu'en conséquence de ces exercices de pénitence, non seulement beaucoup d'entre eux sont tombés malades, mais certains en sont même morts. (Weigle, 1977a, p. 206).

Costumbres crueles en partes retiradas: excès dans la pénitence, rituels tenus secrets, prières non approuvées par la hiérarchie. Des simulations, peutêtre cruelles, de crucifixion de Jésus-Christ. De nouvelles figures rituelles, consacrées à la mort elle-même, que l’on voit désormais monter sur les autels. L'alarme des évêques, certainement justifiée, fut vite suivie par l'intérêt des journaux à scandale de l'est des États-Unis, qui étaient en général d’inspiration plutôt protestante. Des auteurs de tout genre, de foi catholique ou protestante (Chavez, 1954; Darley, 1968; Lummis, 1952; Steele, 1974), ont bâti autour des Penitentes américains une vaste littérature faite de polémiques, d'histoires spectaculaires, d’exagérations et de plaidoyers. Une grande partie de ces écrits, à partir des années 1930, lorsque les Penitentes furent découverts par l’opinion publique américaine, débattent par exemple de la question de savoir si les instruments utilisés pour «crucifier le jeune choisi pour incarner rituellement le Christ» étaient bien des clous ou seulement des cordes. Ou bien s’il avait jamais existé, parmi les Penitentes, une dévotion rituelle adressée en particulier à la mort, qu'on appelait comadre Muerte ou bien la «mort amie»; ou encore si l'exercice de la pénitence témoignait du sadisme des initiateurs du culte ou seulement de leur foi exaltée. Plus récemment, une vision plus équilibrée a émergé, tendant à réfuter les légendes et à faire taire les polémiques; mais elle s’accompagne trop souvent de la défense des dogmes, de la censure sur les témoignages ou les sources embarrassantes, allant jusqu’à requérir des autorités ecclésiastiques l'autorisation de publier des études sur ce thème (Wallis, 1994; Weigle, 1977a).

Nous qui n’avons aucune foi religieuse à défendre ou à condamner, nous opterons ici pour une perspective différente. Nous nous bornerons à revenir à la question que nous avons posée à propos du messianisme apache: qu'est-ce qui a rendu mémorables ces nouveaux rituels et les représentations qu'ils impliquent? Peut-on repérer à l'intérieur de la tradition des Penitentes une cohérence du souvenir, une configuration de traces mnésiques, capables d'éclairer la nature de ce type spécifique d’imagination qui constitue une partie essentielle de la mémoire rituelle néomexicaine et des images qu’elle engen- 
dre? Regardons de plus près les rituels célébrés à l'intérieur de l'Hermandad del Santo Sangre et cherchons à identifier les traits qui permettent de les distinguer d'une simple imitation des cérémonies pratiquées par les confréries espagnoles et européennes.

Nous avons déjà parlé de la situation, définie à la fois par le contact et le conflit culturel, dans laquelle se trouvaient à cette époque les populations indiennes et néomexicaines. Certains auteurs ont cherché à mettre en lumière un échange culturel direct entre Indiens et Espagnols du Nouveau-Mexique. On a pu parler, par exemple, de rituels de flagellation en usage auprès des Pueblo (Bonavides apud Weigle, 1977a, p. 35), de certains Indiens qui seraient devenus membres actifs des Cofradías de Penitentes (Weigle, 1977a, p. 28) ou même, à l'inverse, de la participation de certains Penitentes à des rituels célébrés chez les Pueblo (Rodriguez, 1996). Si on se réfère toutefois à l'état actuel de la recherche, cette piste ne semble pas mener bien loin. La culture des Espagnols du Nouveau-Mexique, et en particulier leurs traditions religieuses (à la seule exception, peut-être, de l'architecture des moradas qui semble souvent reprendre certaines formes hybrides typiques des premières églises construites par les Franciscains avec l'aide des Pueblo), ne semblent pas avoir réagi au contact culturel en cherchant des compromis ou des médiations. Tout semble indiquer, au contraire, que la tradition hispanique a procédé en sens inverse en cherchant constamment à exacerber les différences. L'effort de ces communautés rurales a toujours été de tracer clairement et, ensuite, de conserver jalousement une frontière qui sépare les deux communautés. Cette frontière, à la fois physique et culturelle, a eu pour fonction de maintenir une distinction rigoureuse entre la tradition catholique d'origine européenne et les traditions des tribus amérindiennes avec lesquelles les Néomexicains entraient en contact (McCoy apud Darley, 1968; Rael, 1951; Woodward, 1975). Ce processus a duré longtemps et presque jusqu'à nos jours. Et le premier trait qui frappe l'observateur à propos de ces «nouvelles cérémonies scandaleuses», inventées et pratiquées par les Penitentes, est sans doute leur lien direct avec le conflit qui a longtemps opposé Néomexicains et Indiens. Au sein de cette tradition, on trouve de nombreuses légendes, dans lesquelles l'image du guerrier indien non converti apparaît comme une menace constante de mort. L'Indien non converti (qui peut être, selon les régions, un Comanche, un Pawnee, un Tepehuan et bien souvent un Apache) ne menace pas seulement les fermes, mais aussi les églises fondées par des Hispaniques. Le guerrier indien est un danger direct pour la personne du missionnaire qui, bien souvent, rencontre le 
martyre au cours d'une des attaques régulières livrées par les tribus des Indiens de ces régions. Parfois, les Indiens peuvent aller jusqu'à attaquer l'image même du Christ. C'est ainsi que peut naître un des thèmes récurrents des rituels et des représentations religieuses liés à la tradition des Penitentes néomexicains: celui du Cristo flechado, l'image du Christ sur l'autel transpercé de flèches par les Indiens. Il s'agit là d'un thème précoce et persistant qui caractérise la tradition orale des Penitentes. Du Christ de Mapimi, par exemple (un crucifix vénéré dans les moradas des Penitentes encore au début du siècle dernier), on disait qu'il avait miraculeusement survécu à une attaque livrée par les Tepehuanes contre un sanctuaire catholique du nord du Mexique (Rael, 1951, p. 72-75). Dans l'une des versions de cette légende recueillie dans les années 1930, l'image du Christ sur la croix apparaît comme la cible directe de l'attaque des Tepehuanes qui vise directement l'autel.

[...] C'était un Christ sculpté grand comme un homme. Sous son genou, il y avait une blessure [...] Cette image était l'objet d'une vénération intense. La blessure avait été provoquée par une flèche décochée par les Indiens au cours d'un de leurs assauts au sanctuaire de Mapimi. C'est alors qu'un miracle avait eu lieu: de l'image du Christ avait coulé son sang. (Saravia apud Rael, 1951, p. 72-75).

On peut retrouver les échos directs ou indirects de ce thème, où la présence réelle du Christ et en particulier de son sang semble subitement révélée par ces images, dans un grand nombre d'histoires appartenant à la tradition des Penitentes. Une autre légende, étudiée par W. Wroth (1983), raconte l'histoire du père Juan, un franciscain tué par les Pueblo lors de la rébellion de 1680. Sur le point de mourir, le père Juan prophétisa le retour du Christ et son triomphe dans le Nouveau-Mexique. Dès qu'il fut atteint par une flèche, le ciel se couvrit d'une couleur rouge sombre qui s'appelle encore aujourd'hui, dans toute la région, «sangre de Cristo» (sang du Christ). À cette occasion, le père Juan aurait déclaré: «Tu n’as atteint que mon corps, mais le sang du Christ versé sur ces montagnes va continuer à prêcher le Verbe du Seigneur même après ma mort.» (Wroth, 1983, p. 283-284).

Derrière le corps du franciscain, donc, se cachait la présence du Christ: c'était son sang, et pas seulement celui du père Juan, que les Indiens avaient versé. En témoignait la couleur rouge qui surgit dans le ciel. Le thème du Christ transpercé de flèches n'apparut pas seulement dans des comptes rendus de miracles ou de faits surnaturels; il fut en effet si répandu que, après l’annexion 
du Nouveau-Mexique par les États-Unis, il fut même repris par la presse. En 1891, le Las Vegas Daily Optic publia un article dans lequel on racontait comment des Indiens Apache avaient attaqué des Penitentes qui célébraient une crucifixion simulée; à cette occasion, «le Christ, qu’on avait lié sur la croix, avait reçu des coups de flèches sur tout le corps» (Weigle, 1977a, p. 64).

Ces histoires, et le thème du Christ transpercé de flèches autour duquel elles s'organisent, nous conduisent vers une tradition spécifique qui a élaboré une image rituelle tout à fait particulière de la Passion. Pendant la Semaine sainte, les Penitentes organisaient en effet des processions au cours desquelles les membres de la confrérie devaient imiter la Via Crucis (le chemin de Croix) en traînant sur leurs épaules, tout au long d'un parcours qui allait de la morada à un lieu appelé le Calvaire, d'énormes croix, les maderos. La confrérie avait aussi inventé, sans doute sur la base de représentations de la Passion du Christ liées au théâtre populaire très usuel en Europe, une séquence d'actions cérémonielles au cours de laquelle la Passion était consciemment mimée. Au terme de la procession, un jeune pénitent, la tête cachée par un capuchon, était effectivement lié sur la croix. C'était là un geste qui pouvait, à tout instant, susciter le scandale car ces actions rituelles, qui échappaient, il faut le rappeler, au contrôle de l'Église, suscitaient un grand nombre d'interprétations spontanées, souvent suspectes ou hétérodoxes. On pouvait par exemple avancer l’idée, parfois reprise dans les alabados, louanges ou prières récitées par les Penitentes pendant leurs cérémonies, que le bois sacré du madero et la personne même du Christ devenaient, au cours du rituel, une seule et même chose. Ou encore pouvait-on penser ou affirmer que le Christ lui-même, pendant la procession, se cachait dans le bois de la croix, qui était à son tour identifié à un arbre capable de croître et de donner des fruits - comme dans l'un des alabados recueillis par Rael (1951, p. 204-206):

Sur la croix mon Rédempteur fut cloué à l'aide de trois clous

Le corps de mon Jésus fut ainsi cloué

L'ennemi et la faute l'emprisonnaient

C’est ainsi que le Christ se cache éternellement dans le bois sacré.

Un autre aspect potentiellement dangereux de la crucifixion célébrée par les membres de la Confrérie était certainement la souffrance exagérée infligée au jeune pénitent qui incarnait le Sauveur à l'occasion de la Passion simulée pendant la Semaine sainte. Il est en effet indéniable que le rituel des Penitentes 
tendait à intensifier, au-delà de l'imaginable, l'identification du jeune garçon au Christ de l'Évangile. Une des très rares descriptions de première main que nous avons des célébrations de la Semaine sainte dans une morada souligne cet aspect:

Pendant la nuit de la Porziuncola, un seul pénitent visitait l'église, son corps lié à la croix par une chaîne. En fait, la croix à laquelle il se trouvait lié était presque aussi grande que lui. Le pas du pénitent était donc lent et pénible [...] Ce type de pénitence était sans doute l'un des plus durs. Après que le pénitent était resté lié de cette manière quelques minutes à sa grande croix, sa circulation sanguine ralentissait tellement que les veines de ses bras apparaissaient tendues comme des cordes. Son torse prenait alors une couleur bleu violacé. Bien souvent, le garçon s'évanouissait et tombait par terre [...]. (Wroth, 1991, p. 58-59)

Ainsi le corps du Penitente, dans cet état d'extrême souffrance, prenaitil la couleur bleuâtre qui caractérise souvent l'image du Christ dans l'iconographie de la tradition liée à la Confrérie. D’autres témoignages directs, tel celui rapporté en 1893 par Charles F. Lummis, l'un des rares témoins qui ait réussi à photographier une de ces crucifixions, permettent de compléter cette description et d'approfondir le sens du scandale provoqué par l'excès de ces pénitences:

Je parvins à un accord avec le frère chef, et le jour suivant, le Vendredi saint 30 mars 1888, je pus prendre en photo [...] la procession de ceux qui s'autoflagellaient et de ceux qui portaient des croix bien plus grosses que leur propre corps et trois fois plus longues. Ensuite, le chef de la confrérie, tout en repoussant la foule hostile des spectateurs, marqua sur le sol un endroit où je pouvais poser mon appareil photographique. C'est là, à une distance de quelques mètres, que je pus photographier la crucifixion d'un citoyen américain [...]. Le frère chef entra dans la morada, accompagné de deux de ses assistants. Quelques minutes après, ils en ressortirent en conduisant la victime désignée, un jeune garçon solide habillé seulement de [d'un] pantalon blanc et d'un capuchon noir [...]. Quelques minutes après, le garçon marcha d'un pas ferme jusqu'à la croix qui était posée sur le sol et se coucha complètement sur elle [...]. Plus tard, on apporta une corde bien épaisse, et le Frère de la Lumière commença à ligoter le garçon à la croix. La corde qui était rigide pénétrait profondément dans la chair et bloquait la circulation du sang. En moins de trois minutes, les bras et les jambes étaient noirs [...]. On enveloppa le garçon de la tête aux pieds dans un drap blanc et propre. On ne pouvait plus apercevoir que ses bras, qui avaient une couleur violette [...]. (Lummis apud Darley, 1968, p. 41). 
La simulation de la crucifixion n'était que l'une des souffrances inhumaines que les membres de la Confrérie s'infligeaient. Il y en avait d'autres: à la même occasion, Lummis décrivait des Penitentes qui, au pied de la croix, liaient leurs corps à des cactus, ou même traînaient de lourdes charrettes pleines de pierres sur lesquelles étaient juchées de sinistres représentations de la mort. Nous allons voir ce qu'il faut penser de ces étranges représentations. Arrêtons-nous, pour l'instant, sur l'aspect de ces cérémonies qui suscitait plus que tout le scandale: la douleur physique. Le fidèle de la confrérie, l'Hermano de sangre (le frère de sang), va au-delà des limites de la fiction «théâtrale» à laquelle la représentation du martyre du Christ devait se limiter. Sa souffrance est réelle. Ce qui faisait de cette représentation, aux yeux des Penitentes, un véritable rituel dont on pouvait attendre rédemption et salut, constituait justement, aux yeux de l'Église mais aussi de protestants comme Lummis, un spectacle scandaleux.

Mais le scandale de l’Église avait peut-être encore d'autres raisons. L'exagération du rituel, en insistant sur l'identification du jeune Penitente avec le Christ, finissait par réaliser une identification du jeune souffrant, non seulement avec l'image du Christ, mais aussi avec une véritable représentation de la mort. On pouvait, ainsi, à certaines occasions, voir le Penitente se transformer en squelette, ou bien en défunt revenu du monde des morts. Choisissons, parmi un grand nombre d'exemples possibles, trois images traditionnelles qui illustrent très clairement cette identification réalisée par les Penitentes. La première est une Flagellation du Christ (aujourd’hui conservée dans la collection Bunker) qui vient du nord du Mexique, et que Robert Shalkop a pu relier à la tradition des Penitentes et dater de la fin du XVIII ${ }^{\mathrm{e}}$ siècle. Selon lui (Shalkop, 1970), le thème de la flagellation, très répandu en Amérique, atteint, au Nouveau-Mexique, une intensité qui n'est pas sans faire songer au surréalisme. Dans cette Flagellation, sous la peau lacérée du Sauveur, apparaît en effet l'image d'un véritable squelette.

Une autre illustration de cette tendance à l'identification entre le pénitent, le Christ et la mort nous vient directement d'une morada néomexicaine. Dans cette image, le crucifix se superpose à l'image du velorio, un des instruments principaux des veilles funéraires célébrées par les Penitentes. Pendant ce rituel, les bougies de ce candélabre de forme triangulaire, destiné à la veillée funèbre, étaient éteintes une à une, jusqu'à ce que seule celle qui était située au sommet du triangle restât allumée. Cette bougie, qui symbolisait le Christ, était alors 
transportée dans une pièce voisine, et la morada plongeait complètement dans l'obscurité. À ce moment-là, de la pièce voisine, venaient des sons de maracas et de pito (flûte) et d'autres bruits violents qui symbolisaient le tremblement de terre et le désordre qui, la nuit du Vendredi saint, avaient investi le monde entier à la mort du Christ. Il faut souligner que ce rituel était exclusivement réservé aux cérémonies funèbres destinées à consacrer la mort des membres de la Confrérie.

La croyance selon laquelle les Penitentes entretenaient une relation particulière avec la Mort, qu'ils appelaient «amie» ou comadre est certainement l'un des thèmes constitutifs de la tradition religieuse des Confréries néomexicaines. M. Weigle (1977a) a publié un certain nombre d'histoires, où l'on retrouve régulièrement le thème du Pénitent défunt qui revient parmi les vivants pour participer aux rites de la semaines sainte. Le mort qui revient au cours de la célébration d'un rituel a toujours la tête couverte du capuchon traditionnel, le même que celui que le Christ de la confrérie portait au cours de sa crucifixion simulée. On ne peut donc en rien le distinguer des autres participants au rituel. Pour se rendre compte de sa présence, il faut compter les célébrants, dont le nombre augmente miraculeusement au cours de la cérémonie:

Dans ces temps-là (à la fin du XIX ${ }^{\mathrm{e}}$ siècle), les gens des petits villages croyaient que les âmes des Penitentes morts revêtaient une forme humaine et revenaient dans ce monde pour accompagner les processions en prenant place dans les files formées par les Penitentes [...]. Une femme d'Arroyo Hondo, le Jeudi saint, devait apporter une statue de la Mort provenant de la morada à une église [...]. Deux files de neuf frères l'accompagnaient, mais elle en compta vingt; le dernier de chaque file semblait posséder une sorte de transparence charnelle qui laissait apparaître le dessin du squelette; en particulier les côtes semblaient émerger avec plus de netteté (Weigle, 1977a, p. 182-189).

Dans d'autres histoires, un Penitente encapuchonné, poursuivi par quelques incrédules, enlève de manière inattendue son capuchon et révèle ainsi une tête de mort. C'est ainsi que naît la représentation du Penitente comme un squelette autoflagellant. Cette image se trouve illustrée de manière parlante par notre troisième exemple, une petite sculpture publiée par M. Weigle (1977b) et aujourd'hui conservée au musée de Denver. Une figurine représentant un squelette est agenouillée devant une croix; entre la croix et le squelette en prière se trouve une petite tête de mort. 
Dans les temps de crise, comme au cours de cette année 1886, où l'hiver fut si rigoureux que beaucoup de pasteurs et de paysans moururent littéralement de froid, on raconte que des groupes entiers de Penitentes défunts s'unirent aux vivants pour célébrer le rituel de la Semaine sainte et implorer, ainsi, avec eux, l'aide du ciel. La morada elle-même, émergeant alors dans une autre lumière, apparaissait comme la résidence des frères de l'autre monde (Hermanos del Otro Mundo). C'est peut-être là le thème qui rapproche le plus les Penitentes des chamanes apache, comme Noch-ay-del-klinne, Big John ou Daslahdn, qui, pour réagir à leur défaite et à l'invasion des Blancs, cherchaient désespérément sur la tombe des chefs indiens qui venaient de mourir, à rétablir un contact rituel avec leurs morts. Dans des villages hispaniques comme Cordova ou Arroyo Hondo, les tinieblas, les rituels funéraires que les Penitentes célébraient dans l'obscurité de la morada, avaient précisément ce but:

Les frères Penitentes et les habitants du village s'adossent littéralement les uns aux autres, en s'immergeant dans cette obscurité où résonnent les bruits du désordre du monde qui a investi la terre après la Passion du Christ ils appellent, ensemble, les noms de leurs défunts, les noms de ceux qui ont construit la communauté par le passé. (Wroth, 1991, p. 62).

Dans cette perspective, la morada construite et entretenue par la confrérie des Hermanos apparaît comme un lieu très différent de l'église, précisément parce qu'elle se veut une «maison des morts». Un autre alabado, recueilli par Alice Corbin Henderson en 1937, où le récitant incarne la voix du défunt, confirme cet aspect essentiel des croyances des Penitentes (Boyd, 1974, p. 121):

Adieu pour la dernière fois

qu'on me voit sur la terre

On me donne sépulture

c'est là ma vraie maison

Adieu à tous ceux qui sont présents

à tous ceux qui m’accompagnent.

Nous avons vu que la réaction de l’Église catholique à la diffusion de ces rituels et de ces croyances, une réaction dont nous commençons à comprendre les raisons, fut rapide et énergique. Déjà, l'évêque Zubiria parlait de carnicerias (de «carnages»). Toutefois, ce n'était pas seulement l'effort physique, très lourd, 
demandé par la pénitence cruelle qui conduisait le Clergé à parler d' 'excès»: il y avait des faits et des actions encore plus graves. Parmi les rituels célébrés dans les moradas, qui incluaient des processions tout à fait traditionnelles comme celle qui mettait en scène la rencontre (Encuentro) entre la Sainte Vierge et le Christ, on trouvait aussi des célébrations moins acceptables, des histoires qu'on racontait à propos des défunts et de leurs squelettes encapuchonnés. Une de ces cérémonies était le transport d'une étrange représentation de la mort, appelée dame Sébastienne (Doña Sebastiana), qui, dans une atmosphère de grande dévotion (Weigle, 1977b, p. 144), était apportée jusqu'à l'autel de la morada, où elle prenait sa place à côté des images des saints, de la Vierge et de JésusChrist lui-même (fig. 1 et 2). Dans d'autres occasions, mais en particulier au soir du Vendredi saint, cette figure posée sur une charrette remplie de pierres était traînée par un fidèle jusqu'à la Croix. Ici, l'action rituelle assumait presque la forme, bien que rapide et rudimentaire, d'un triomphe. En fait, cette figure énigmatique et ses actions suscitaient un certain nombre de croyances. Une de ces croyances rappelle nettement le thème du Christ transpercé par les flèches:

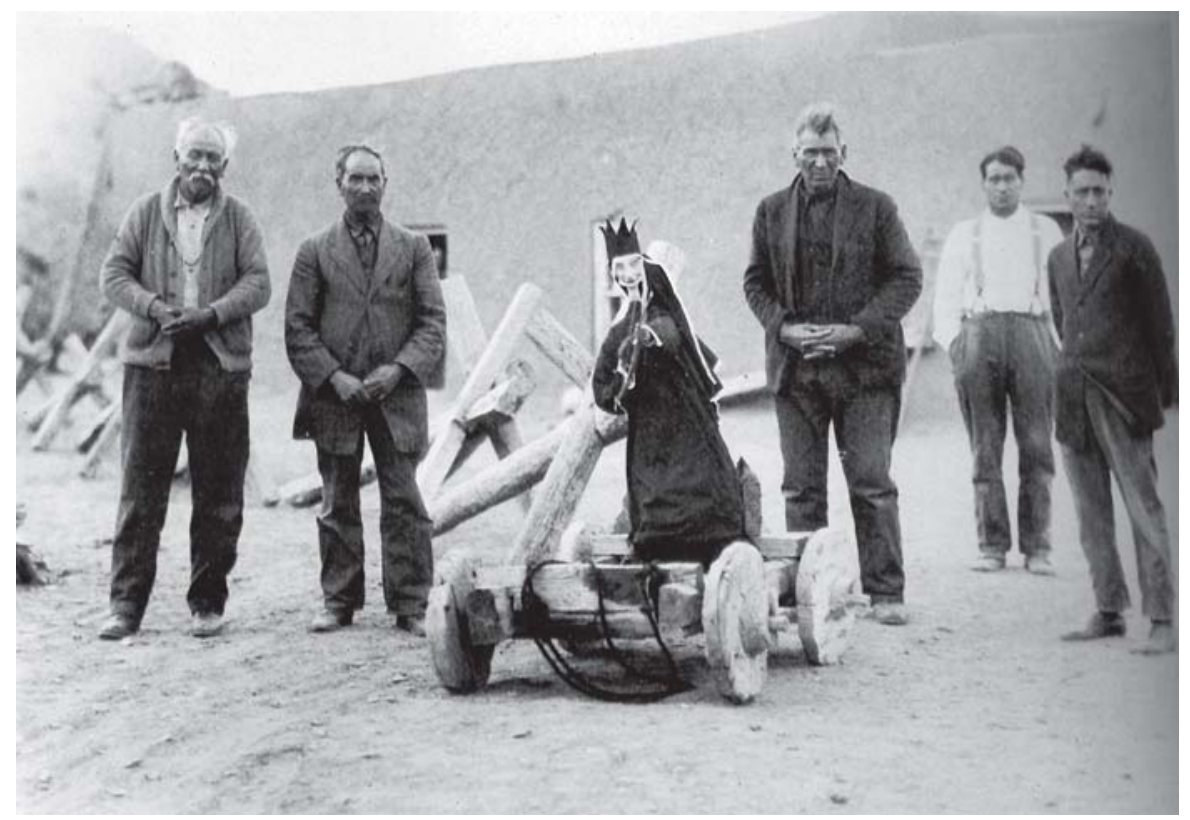

Figure 1.

Horizontes Antropológicos, Porto Alegre, ano 14, n. 29, p. 43-66, jan./jun. 2008 


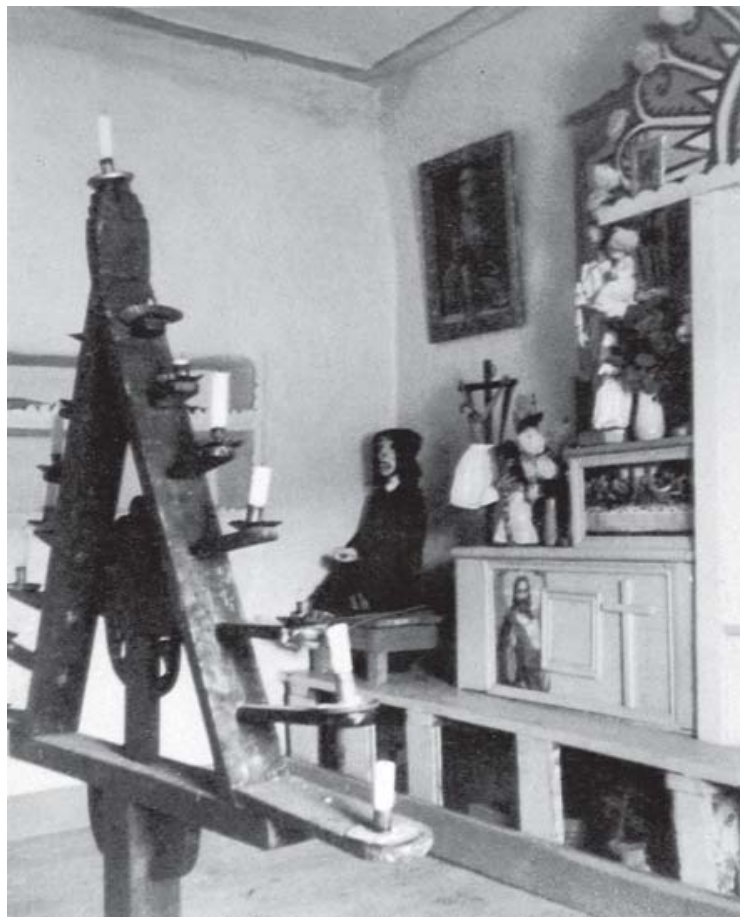

Figure 2.

on croyait et on racontait que dame Sébastienne avait une fois (précisément comme les Apache, ainsi que l'avait raconté le Daily Optic de Las Vegas) tué un des Penitentes, alors qu'il se trouvait sur la croix, en le transperçant d'une flèche. La figure de la Mort démontrait ainsi, d'un côté, son effroyable efficacité, de l'autre, le fait que derrière la sculpture qui la représentait, se trouvait la présence bien réelle d'une mort. À cette légende qui lie cette figure à l'histoire du Christ de Mapimi et d'autres dont nous avons aussi parlé, correspondait en effet un geste rituel très éloquent. Pendant l'une des cérémonies secrètes célébrées dans la morada, c'était dame Sébastienne qui choisissait le jeune garçon qui devait rituellement incarner le Christ sur la croix. On affirmait alors que c'était la statue elle-même, parfois représentée comme une femme aveugle, parfois au contraire dotée d'un regard menaçant lancé par des yeux faits de fragments de verre ou de métal, qui décochait une flèche contre le jeune garçon à partir de l'autel. Ce geste le désignait comme digne du nom qu'il allait porter pendant une année entière, il était ainsi devenu grâce au geste de dame Sébastienne «el Cristo» de la confrérie.

Derrière cette représentation intimement liée à l'image du Christ, mais aussi à celle de l'Indien agresseur, se cache une logique complexe qui implique à la fois le plan de l'iconographie et celui de l'action rituelle. Considérons, tout d'abord, l'exemple peut-être le plus ancien de la représentation de dame Sébastienne. Il s'agit d'une sculpture commandée à Nazario Lopez de Cordova par la morada de Las Trampas, et qui fut réalisée presque certainement en l’année 1860 (Boyd, 1974). Cette sculpture appelée aujourd’hui Muerte en su 
carreta est conservée au musée du Nouveau-Mexique d’Albuquerque (fig. 3). John Bourke, ce même officier de l'armée des États-Unis qui, pour y avoir assisté, put décrire des rituels apache consacrés à la vénération des croix portant l'image d'un serpent et des danses de Gan, vit la Muerte en su carreta en 1881. En fait, la désapprobation, à la fois protestante, laïque et rationaliste, qui anime la réaction du jeune Bourke est analogue à celle qu'il va manifester, quelques années plus tard, en 1891, face aux danses des Apache:

Bourke se trouve dans une église. Dans une pièce située à droite de la porte principale et qui correspondait à notre sacristie, il y avait une horrible statue habillée en noir, au visage blême. Elle portait un capuchon de moine et tenait en main un arc où une flèche se trouvait déjà en position de tir. Cette figure était assise sur une sorte de charrette en bois, semblable à un petit char d'artillerie, mais construite du bois le plus rudimentaire et composé de quelques pièces assemblées par de gros clous. Les roues étaient des sections de tronc de pin, la barre et le châssis non huilés faisaient un bruit désagréable, et à la difficulté de pousser ce véhicule s'adjoignait le fait que le siège sur lequel la Mort était assise tel un cocher lugubre était rempli de pierres de formes irrégulières. (Bourke apud Weigle, 1977b, p. 142).

Cette représentation était exposée, jusqu'aux années 1960, dans l'église de San José, avec trois crânes dont on disait qu'ils appartenaient à trois missionnaires tués par des Indiens. Dans la sculpture de Nazario Lopez, directement liée aux cérémonies célébrées par la confrérie, la tête de dame Sébastienne est une tête de mort; son corps composé de branches d'arbre est couvert d'un grand manteau. Dame Sébastienne tient à la main un arc et des flèches. L'ensemble de la figure est posé sur une charrette analogue, sous une forme

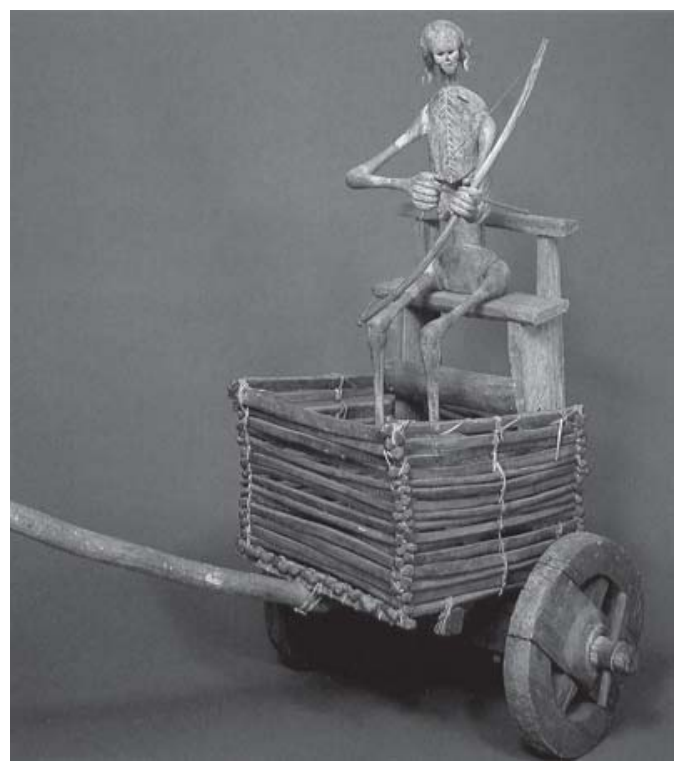

Figure 3. 


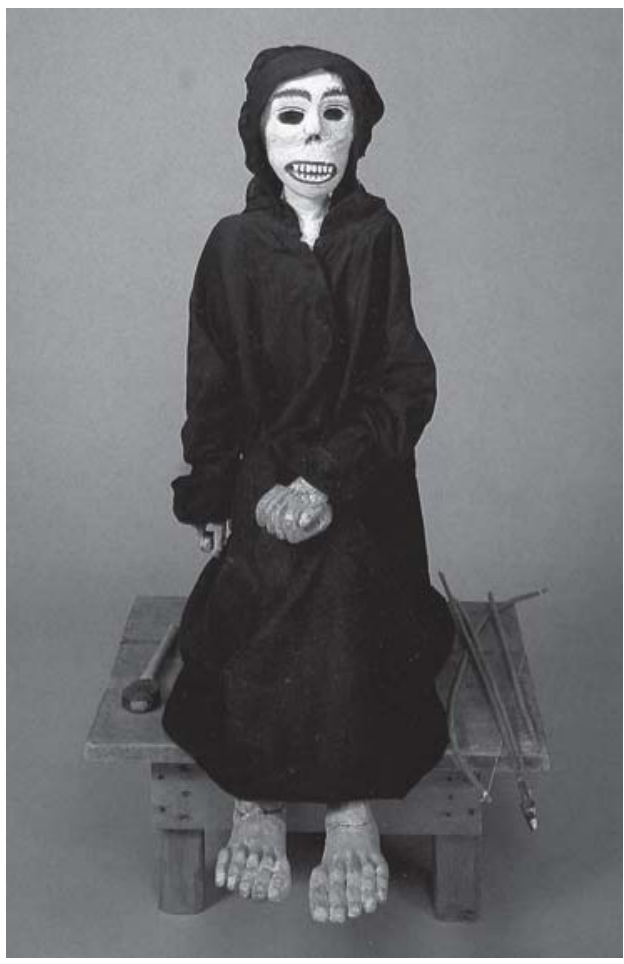

Figure 4. rudimentaire, au char figurant dans les représentations des Triomphes de la Mort qui ont eu une fortune considérable dans le Moyen Âge hispanique et européen. Ce premier modèle iconographique, pendant tout le $\mathrm{XIX}^{\mathrm{e}}$ siècle, se développe entre le nord du Nouveau-Mexique et le sud du Colorado dans deux directions ainsi que l'analyse l'historienne de l'art Louise Stark. On peut en effet distinguer entre deux types de représentation de dame Sébastienne: le type «Sangre de Cristo» et le type "Vallée de Saint-Louis». Les représentations originaires du Nouveau-Mexique sont, en général, des «squelettes assis ou agenouillés» qui portent un arc et des flèches. Ces figures, dites «Sangre de Cristo», sont caractérisées par

de grandes mains, une tête de petite dimension, d'aspect grotesque; souvent quelques mèches de cheveux gris ou blancs sont fixées sur la tête en bois [...] D'autres fois, on applique aussi à la tête des "yeux" d'obsidienne, mais bien souvent les deux orbites restent vides. Une attention soutenue est réservée à l'anatomie du corps sculpté en bois: en général, les côtes proéminentes et les membres n'ont pas les proportions normales. (Stark, 1971, p. 305).

Les représentations originaires de la vallée de Saint-Louis (fig. 4) sont assez différentes:

La figure est souvent assise sur une charrette, elle porte un arc et des flèches; le corps n'est pas entièrement sculpté, il est plutôt composé de blocs de bois et couvert d'un manteau sombre. De la tête, on ne sculpte que le visage, le reste étant recouvert d'un capuchon qui couvre à moitié les yeux et leur confère une expression menaçante [...]. (Stark, 1971, p. 305). 
Le petit groupe de sculpteurs hispano-américains proche de la tradition rituelle des Penitentes réélabore donc, dans des conditions complètement nouvelles et dans le contexte d'un art populaire, un thème iconographique d'origine médiévale. Ce thème s'est développé en Europe de plusieurs manières, qui vont de la Danse des morts, répandue en Europe centrale, jusqu'aux différentes formes prises par la Carreta de la Muerte (la charrette de la Mort), typique de la tradition espagnole. Nous ne pouvons naturellement pas retracer, ici, l'histoire de la survivance américaine de cet illustre thème iconographique européen (Camille, 1996; Frugoni, 1988); il suffira d'en examiner brièvement certains traits et d'en comprendre le développement, dans le cadre de cette véritable renaissance iconographique qui s'est réalisée dans l'art populaire du Nouveau-Mexique tout au long du XIX ${ }^{\mathrm{e}}$ siècle. Dame Sébastienne est certainement le fruit d'une tradition nouvelle, dont on peut identifier avec une certaine précision les origines au Nouveau-Mexique. Mais elle est aussi le résultat d'un dernier retour à une tradition iconographique chrétienne très ancienne à laquelle se réfère le groupe de sculpteurs et peintres néomexicains qui sont directement liés à la construction et à la décoration des moradas. Ces artistes sont à l'origine d'une tradition que l'on appelle «l'art des santeros». Comme l'a bien vu George Kubler (1964), ces santeros du Nouveau-Mexique reprennent en général, avec l'attitude conservatrice extrême qui est typique des populations isolées, l'iconographie traditionnelle de l'Église catholique, bien qu'ils n'en connaissent presque jamais les sources illustres. Chez un artiste comme l'auteur anonyme de la planche conservée au musée Taylor d'Alice Springs, on trouve par exemple une représentation de la Trinité réalisée à travers la réplique de trois figures simplement esquissées mais identiques. Ce thème iconographique, d'origine byzantine, avait été interdit dès 1745 par le pape Benoît XIV. Il s'agit donc d’une tradition figurative, celle des santeros, qui est pleine d'archaïsmes et de répliques provinciales. Cette tradition liée à la Confrérie des Pénitents est toutefois aussi une tradition nouvelle. Comme Kubler l'a remarqué, l'attitude conservatrice des santeros néomexicains s'accompagne de procédés tout à fait caractéristiques de cette tradition; il s'agit notamment de la fusion de thèmes iconographiques différents en une seule image. De ce processus nous avons d'ailleurs vu un premier exemple: l'image du Calvario, qui se trouve engendrée par la superposition du crucifix et du velorio (le chandelier triangulaire utilisé pour la veillée funèbre). Un processus analogue est tout à fait repérable dans le cas de la représentation de Nuestra Señora de los Dolores (Notre Dame des Douleurs), une représentation qui se trouve fondue avec celle de sainte Véronique (Wroth, 1991). 
En même temps que cette contamination iconographique opère certainement ce que Warburg a appelé le processus de polarisation des images. L'action rituelle confère à l'iconographie, dans cette tradition, une dynamique nouvelle à partir de laquelle naissent de nouvelles significations et de nouveaux rapports entre images. Cela apparaît de manière particulièrement claire dans l'usage rituel des représentations sculptées des saints et de la Vierge. Voyons encore un exemple. Le fait que dame Sébastienne se trouve habillée d'un grand manteau noir, surtout dans les représentations du type «Saint-Louis», peut faire penser à une contamination possible avec le thème iconographique de Notre Dame des Douleurs. Les deux images se trouvent associées à la même fonction de représentation du deuil. La différence entre les deux personnages peut paraître banale: alors que Notre Dame des Douleurs subit la souffrance, dame Sébastienne, telle que nous la voyons dans la procession des Penitentes au cours de la Semaine sainte, est là pour la donner à d'autres. Porter son image sur les épaules signifie bien souvent vouloir expier ses péchés. Toutefois, l'usage rituel de l'image (par l'une de ces transformations «polaires» qui opèrent dans cette dimension que Warburg a appelée «la vie posthume des iconographies») peut en changer le sens, transformer une représentation passive en une représentation active. Dans le contexte des pénitences, ce passage concerne la figure de Notre Dame des Douleurs et en révèle par là même l'ambiguïté. Laurence F. Lee, l'un des premiers témoins des rituels de la confrérie, décrit ainsi l'une de ces cérémonies:

L’image de Notre Dame des Douleurs était parfois utilisée comme moyen de pénitence. On dit qu'un membre de la confrérie portait cette image sur sa tête en levant bien haut les bras. Le garçon aurait-il baissé les bras que les lames de deux longs couteaux insérés à la base de la sculpture en bois auraient aussitôt pénétré sa chair. (Lee, 1920, p. 10).

Cette logique de la contamination en une seule représentation d'images différentes et contradictoires, particulièrement intense dans le cas de dame Sébastienne, ne se limite toutefois pas à cet aspect. Le processus qui semble gouverner la survivance du thème européen du Triomphe de la Mort sous le trait de dame Sébastienne en Amérique implique, au moins, trois aspects: la contamination iconographique, la polarisation des significations rituelles (actifpassif) et finalement, en tant que résultat des deux opérations précédentes, une grande intensification de l'image qui devient ainsi particulièrement saillante. Ce 
caractère, qui la rend à la fois inattendue et complexe, peut rendre compte de sa diffusion et de sa pérennité. La contamination joue ici (comme dans le cas de Silas John qui, de chamane qu'il était, se proclame «Nouveau Christ») dans le contexte de la confrontation et du conflit culturels. Dame Sébastienne, certes, représente la Mort; toutefois elle ne porte pas - comme d'autres représentations européennes ou mexicaines qui en constituent probablement la source immédiate - une faux pour cueillir les âmes. Au contraire, elle porte un arc et des flèches, un thème iconographique complètement inconnu dans les terres d'origine de la culture néomexicaine, c'est-à-dire le Mexique et l'Espagne. Très rares en Europe, ces attributs de la Mort étant entièrement cohérents avec le thème américain, lié à la prédication franciscaine, du Christ percé de flèches. Aussi cette commère menaçante n'est-elle pas, comme ses funèbres sœurs mexicaines et espagnoles, seulement «une Mort». Pour la confrérie des Penitentes du Nouveau-Mexique, elle s'appelle aussi «Sébastienne», "commère Sébastienne». Un des modèles iconographiques et religieux auquel cette étrange représentation est indirectement associée est donc certainement un saint Sébastien (et derrière Sébastien peut-être, comme nous le verrons plus tard, apparaîtra un Christ flagellé à la colonne). Cette relation singulière avec la figure de Sébastien le martyr, que certains auteurs ont bien hâtivement attribuée à une pure et simple «confusion» (Boyd, 1974; Stark, 1971; Weigle, 1977a) est certainement paradoxale et demande une explication. Dans un essai détaillé, et tout à fait fascinant, William Wroth (1991) a reconstruit attentivement les origines des images funéraires des Penitentes et des objets rituels qui les accompagnent. Sa conclusion en ce qui concerne dame Sébastienne est qu'une dérivation de l'iconographie catholique mexicaine est hors de doute: ces Muertes viennent de l'Europe et constituent naturellement la manifestation ultime d'une série qui a son origine dans les Triomphes du Moyen Âge. Une des étapes de ce processus de métamorphose de la Mort en Triomphe a peut-être été la représentation des Tarots ${ }^{1}$. Quoi qu'il en soit, cette Mort en Triomphe atteint le culte de la Passion du Christ pendant la Semaine Sainte, tel qu'il était pratiqué au Nouveau-Mexique par les confréries du Saint Sang. Selon Wroth (1991), le rite catholique traditionnel auquel se réfère cette représentation est la cérémonie

1 Selon une thèse chère à Boyd, qui a été récemment contestée par M. Weigle (voir Weigle, 1977a, p. 169, 271, note 48). 
mettant en scène la sépulture du Christ au soir du Vendredi saint, dans lequel celui-ci est représenté dans l'acte de triompher rituellement sur la mort. Celleci est figurée au pied de la Croix, accompagnée d'expressions comme «Mort, où est donc ton triomphe?» ou bien «Mort, je serai ta mort.» L. Stark a reconstruit une série tout à fait vraisemblable de transformations iconographiques qui mènent de Séville au Nouveau-Mexique, en passant par des iconographies mexicaines, comme celle liée au Paso funéraire, en usage dans la ville de Mexico déjà à partir du XVI siècle (fig. 5).

Ce n'est donc pas le contexte qui nous étonne dans les représentations néomexicaines de la mort, mais plutôt, dans l'apparition de dame Sébastienne, d'autres aspects qui la rendent tout à fait singulière et en modifient fortement la signification traditionnelle. Avant tout, il faut remarquer qu'en triomphant sur sa
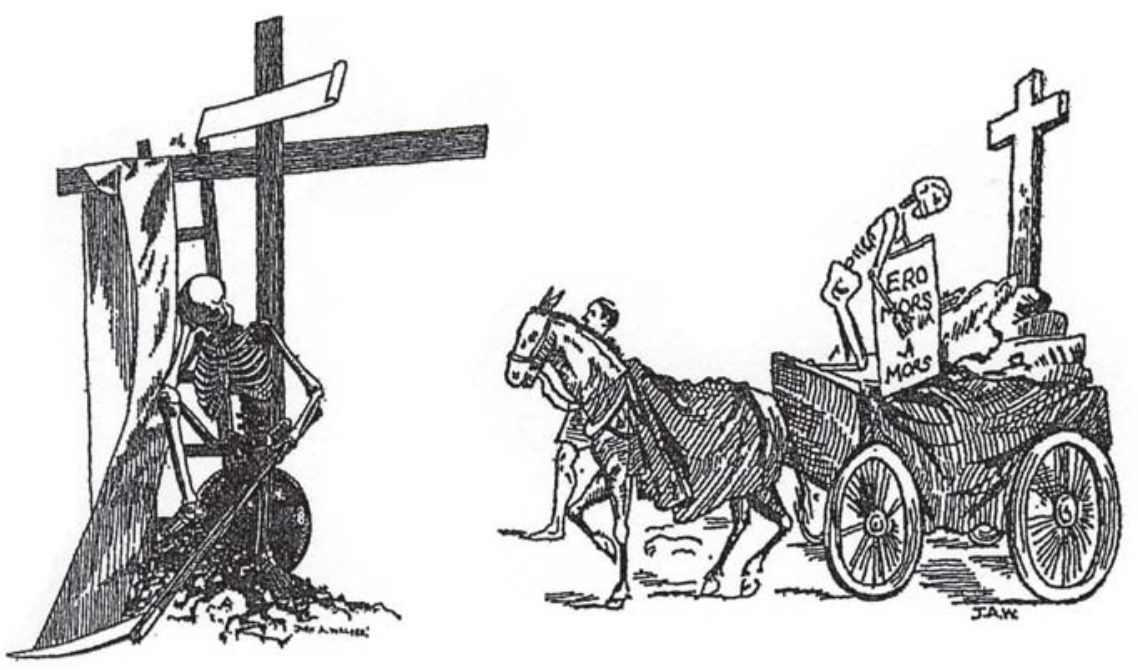

Figure 5.

charrette traînée péniblement par le Pénitent, dame Sébastienne témoigne d’une inversion du rituel consacré au triomphe de Jésus-Christ. Au sein de la mora$d a$, en suivant une logique de l'action rituelle que Victor Turner a appelée «transitionnelle», ce n'est donc pas le Christ qui triomphe sur la mort. C’est plutôt elle, Sébastienne, qui, en décochant ses flèches contre Lui, triomphe sur le Pénitent identifié à la personne du Christ; c’est elle que le Pénitent doit traîner en triomphe 
sur une charrette chargée de pierres. L'autre aspect surprenant concerne l'iconographie. Dans la très grande majorité des cas, à la place de la faux traditionnelle, nous voyons apparaître l'arc muni de flèches qui met immédiatement la représentation de la mort en relation avec l'image menaçante de l'Indien des légendes des Penitentes liées au thème du Christ percé d’une flèche. Dans le même sens va l'invention du nom de cette représentation rituelle qui, de simple Muerte en su carreta (Mort sur sa charrette) comme elle est encore appelée dans le Don Quichotte de Cervantès, devient dame Sébastienne. Par cette nouvelle dénomination, la représentation de la Mort se trouve symboliquement reliée à un saint Sébastien frappé par des flèches, une représentation qui reste pour ainsi dire en arrière-plan, évoquée mais non représentée.

Cette métamorphose implique une deuxième inversion: dame Sébastienne décoche ses flèches, elle n'en reçoit pas comme le saint lié à la colonne. Dans la logique de la célébration rituelle, l'apparition de saint Sébastien ouvre ensuite une série ultérieure de connotations analogiques, celles qui mènent au Christ à la colonne dont parlent les hymnes chantées par les Penitentes pendant la procession (Weigle apud W. Wroth, 1991, p. 61):

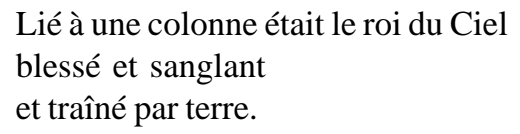

Dans l’Évangile, le Christ, comme Sébastien, est lié à une colonne. Dans l’image simulée de son martyre, telle qu'elle apparaît sur la scène du rituel célébré par les Penitentes, il est, comme Sébastien, atteint par la flèche décochée par dame Sébastienne. Cette séquence d’images, qui révèle une série d’identifications rituelles partielles, désigne le personnage complexe qui se trouve incarné par le Penitente au cours du rituel. Celui-ci, d'un côté, reçoit la flèche, comme Sébastien, et de l'autre côté, verse à l'imitation du Christ son sang dans le martyre. Rappelons que, en tant que Hermano de sangre (frère de sang), c'est au sang du Christ qu'il a promis sa dévotion. Le sang qu'il verse en se flagellant devient, pendant le rituel de la crucifixion simulée, «le» sang du Christ. Le Pénitent pousse l'identification jusqu'à porter le poids de la croix sur ses épaules comme le Christ, mais, au-delà, jusqu’à monter lui-même sur la croix du martyre.

Dame Sébastienne ne peut donc se comprendre que si on l'insère dans deux séries de représentations. D’un côté, elle renvoie à une série complexe 
d'identifications partielles à la figure de sa victime rituelle, le «Christ» de la Confrérie. D’autre part, elle renvoie aussi, par la flèche qu'elle décoche, à la présence de l'Indien en tant qu'ennemi. Sa représentation réalise ainsi, simultanément, deux opérations propres au rituel. La première inverse le rite traditionnel en transformant le triomphe du Christ sur la mort en un triomphe de la mort sur un Christ rituellement représenté par le Penitente; la seconde, en évoquant la flèche de l'Indien en tant que véhicule d'agression symbolique à l'égard du Christ, insère dans le nouveau rituel la trace d'un conflit entre cultures différentes.

Face au Christ incarné par le Penitente (et partiellement identifié avec Sébastien mais aussi, comme on l'a vu, avec un membre de la communauté des morts), se trouve une figure apparemment traditionnelle, en grande partie construite à partir des éléments symboliques préexistants. Néanmoins, cette figure témoigne d'un usage si nouveau, si contradictoire et paradoxal de ces éléments, qu'elle en devient un moyen d'indiquer la présence d'une figure étrangère au système de représentation traditionel: celle de l’Indien.

Rappelons ensuite que Sébastienne, tout en provenant du culte traditionnel de la Semaine sainte, témoigne d'une inversion de ce rituel. Si nous parcourons la série d'identifications partielles dont elle se trouve composée, une opération que les croyants se gardent bien de réaliser, nous comprenons que c'est bien elle qui triomphe sur le Christ, et non pas le contraire, comme le voulait la tradition officielle. Ce dernier aspect rend sa représentation particulièrement inquiétante et en fait le véhicule d'une présence étrangère au rituel catholique. Dame Sébastienne a une nécessité propre à l'intérieur de ces cérémonies. C'est à travers elle que l'Indien, une présence bien réelle, une menace quotidienne, peut entrer dans le lexique symbolique de la communauté religieuse des Penitentes. C’est de là que naissent peut-être, parmi les observateurs extérieurs de culture différente, comme l'évêque Lamy et les anglo-protestants, ce vague sentiment de sacrilège et, surtout, cette accusation, qui peut paraître étrange à première vue, de partager avec les Indiens un mystérieux culte de la mort. L'Indien agresseur n'est pas montré en tant que tel, mais il fait son entrée dans l'église par le biais de son arc et de ses flèches. Il s’agit d'un détail qui peut sembler insignifiant, mais qui indique la partie implicite, très claire du point de vue de l'analyse autant que vigoureusement refoulée par le croyant croyant, de l’image néomexicaine de la mort.

Cette présence implicite de l'Indien qui porte la mort fait de dame Sébastienne l'image intense d'un conflit non résolu tout autant que le vecteur 
collectif d'un oubli. Un double oubli, pourrait-on dire. D’un côté, celui de la signification originale du rituel chrétien de la Semaine sainte, où le Christ triomphe de la mort, qui est ici d'abord oubliée puis inversée. Et de l'autre, l'oubli en tant que déni du conflit présent avec les Indiens, lié au refus du contact et à l'exaspération des différences culturelles qui traversent toute la tradition religieuse des Penitentes. "Ce ne sont pas eux, les étrangers, semble déclarer la statue de dame Sébastienne, qui menacent notre vie; c'est notre mort qui arrive à travers eux. C'est la mort que nous connaissons, telle que notre Église la représente depuis toujours.» Sauf que, précisément, un détail a changé: cette flèche décochée, qui immerge toute la représentation dans l'ambivalence et en fait une image extrême de la relation fragile qui peut s'établir au cœur même de l'action rituelle, entre mémoire et oubli.

\section{Réferences}

BOYD, E. Popular arts in Spanish New Mexico. Santa Fé: Museum of New Mexico Press, 1974.

CAMILLE, M. The master of death. Oxford: Oxford University Press, 1996. CHAVEZ, A. The Penitentes of New Mexico. New Mexico Historical Review, v. 29, p. 97-123, 1954.

DARLEY, Alex M. The passionists of the southwest or the holy brotherhood. Rio Grande Press, 1968.

ESCUDERO, Jose Agustin de. Noticias historicas y estadisticas de la antigua provincia del Nuevo-Mexico. Las Vegas: Las Vegas Press, 1971.

FRUGONI, C. Altri luoghi, cercando il Paradiso: il ciclo di Buffalmacco nel Camposanto di Pisa. Annali della Scuola Normale Superiore, Pisa, v. 18, n. 4, p.1557-1643, 1988.

KUBLER, G. Santos: folk art of New Mexico. Fort Worth : Amon Carter Museum, 1964.

LEE, L. F. Los hermanos Penitentes. El Palacio, v. 8, n. 1, 1920.

LUMMIS, Charles F. The Land of Poco Tiempo Albuquerque: University of New Mexico Press, 1952. 
RAEL, Juan B. The new mexican alabado. Stanford: Stanford University Press, 1951.

RIEUPEYROUT, J.-L. Histoire des Apache. Paris: Albin Michel, 1987.

RODRIGUEZ, S. The Matachines dance: ritual symbolism and interethnic relations in the Upper Rio Grande Valley. Albuquerque: Universitiy of New Mexico Press, 1996.

SHALKOP, R. Spanish colonial painting. Colorado Springs: Taylor Museum, 1970.

STARK, L. The origin of the Penitente "Death Cart". Journal of American Folklore, v. 84, 1971, p. 304-311.

STEELE, Thomas J., S.J. Santos and saints: essays and handbook. Albuquerque: Calvin Horn, 1974.

WALLIS, M. En divina luz: the penitente moradas of New Mexico. Albuquerque: New Mexico University Press, 1994.

WEIGLE, M. Brothers of light, brothers of blood: the penitentes of the Southwest. The Western Historical Quarterly, v. 8, n. 1, p. 61-62, Jan. 1977a.

WEIGLE, M. Ghostly flagellants and Dona Sebastiana: two legends of the Penitente Brotherhood. Western Folklore, v. 36, n. 2, p. 135-147, Apr. 1977b.

WEIGLE, M.; LYONS, T. R. The celebration of community in Penitente villages. In: TURNER, Victor (Ed.). Celebration: studies in festivity and ritual. Washington D.C.: Smithsonian Institution Press, 1982. p. 231-251.

WOODWARD, Dorothy. The penitentes of New Mexico. PhD diss., Yale University, 1975.

WROTH, William. La sangre de Cristo: History and symbolism. In: WEIGLE, M. (Ed.). Hispanic Arts and Ethnohistory in the Southwest. Albuquerque: University of New Mexico Press, 1983. p. 283-292.

WROTH, William. Images of penance, images of mercy: southwestern santos in the late nineteenth century. Norman: University of Oklahoma Press, 1991. 